\title{
Explore the Application of Electron Work Function in Material Design towards "Electronic Metallurgy"
}

\author{
D.Y. Li \\ Department of Chemical and Materials Engineering \\ University of Alberta \\ Edmonton, Alberta, Canada T6G 1H9
}

\begin{abstract}
With rapid advance in material technology, material design has been required to rely on more fundamental principles. Significant efforts have long been made to correlate properties of materials to their electron state based on quantum mechanics. However, quantum theories are generally complicated and unfeasible for material design, especially for structural materials which generally have multiphase microstructures. It is thus highly desired to have simple but fundamental parameters, which reflect the electron behaviour of materials, for material design in a feasible manner. In this talk, electron work function, which is the minimum energy to move electrons at Fermi level inside a solid to its surface, will be demonstrated to be a promising parameter to characterize materials and provide supplementary clues for material design and modification.
\end{abstract}

